\title{
Semantic SPA framework for situational student-project allocation in education
}

\section{Shreyas Suresh Rao}

\author{
Department of Computer Science and Engineering, \\ School of Engineering, \\ Presidency University, India \\ Email: shreyas@presidencyuniversity.in
}

\begin{abstract}
Web 3.0 technologies have redefined the way classical problems in education are solved. The classical problems are constrained by interoperability challenges that can be addressed using semantic technologies. Student-project allocation (SPA) is one such classical problem in education, whose solution lies in finding a stable matching between student-project pairs. Although many methods exist to solve the SPA problem, recent developments in higher education, such as globalisation, has triggered interoperability challenge because of diversity in student and expert availability across disciplines, campuses, and universities. The author proposes the semantic SPA framework that addresses the interoperability challenge, by leveraging the existing semantic investments made by the educational institutions. The framework standardises the SPA data elements formally using semantic Web concepts, proposes reference ontology for the situational SPA problem, and adopts a semantic Web service approach, using OWL-S, for development. The OWL-S specification enables the automatic discovery, composition, and invocation of the Web services by educational Institutions or semantic agents on the Web, for situational SPA scenarios.
\end{abstract}

Keywords: semantic web services; student-project allocation; SPA; semantic interoperability; Web 3.0.

Reference to this paper should be made as follows: Rao, S.S. (2020) 'Semantic SPA framework for situational student-project allocation in education', Int. J. Intelligence and Sustainable Computing, Vol. 1, No. 1, pp.69-82.

Biographical notes: Shreyas Suresh Rao is an Assistant Professor in the Department of Computer Science and Engineering at Presidency University, Bengaluru, India. He obtained his PhD from Manipal Institute of Technology, Manipal Academy of Higher Education, India. His previous stint includes working in academia for five years and working in the IT industry for seven years. In his IT stint, he played various roles such as Business Analyst, Team Leader, Analyst/Designer, and had been involved in the execution of several end-to-end enterprise projects in banking, manufacturing and automobile domains. His technical expertise lies in ASP.NET technologies such as Web services, Web applications, Silverlight, and SharePoint. His research interests include semantic web, linked open data, crowdsourcing, knowledge engineering and web services. 


\section{Introduction}

Evolution of the World Wide Web has transformed education significantly in the past two decades (Devedzic, 2006). The earliest version of the Web, known as Web 1.0, focused on information sharing among the users through the creation of Web applications. In the educational domain, Web 1.0 facilitated experts to create educational websites, post videos, and other e-learning material on the Web, and communicate to students in a non-interactive mode (Liu and Kender, 2004). Gradually, by early 2000, the Web transformed into a collaborative medium, known as Web 2.0, which allowed the users to contribute content to the Web and interact with one another through Web applications such as Twitter, YouTube, Facebook, etc. (Murugesan, 2007). In the educational context, these applications facilitated collaboration among inter-student community as well as student-teacher community (Solemon et al., 2013) by empowering non-technical users to contribute content to the Web, which was earlier the privilege of only technical users. Although Web 2.0 technologies generated massive amount of data through user collaboration, there was no mechanism to identify the context in which the users contributed. This limitation is overcome by the creation of domain-based shared ontologies, which provide contextual description for all data elements through the Semantic Web or Web 3.0 technology (Shadbolt et al., 2006). The Web 3.0 technology is used in education to establish semantic interoperability and facilitate data sharing across educational boundaries (Yu et al., 2012).

Embracing the semantic bandwagon, educational institutions are investing heavily in semantic technologies by converting their institutional data into the semantic format. A sample list of Universities exposing their institutional data as linked open data (LOD) include the University of Bristol, The Open University, Ege University, to name a few (Linked Universities, 2014). Among these, some universities have exposed their expert (researcher) data as LOD, while others have exposed their student data as well. As a consequence of this semantic investment, various facets of education such as e-learning (Millard et al., 2008; Ghaleb et al., 2006), teacher education (Czerkawski, 2014), and offer selection process (Kumar et al., 2009), have been redefined semantically, in order to facilitate interoperability among the educational institutions.

The student-project allocation (SPA) is a classical problem in education that needs a similar semantic overhaul. Conventionally, in universities, experts offer projects to students as part of final-year projects (FYPs) (Teo and Ho, 1998). These projects are planned well in advance, and allocated, based on expert and project capacity constraints, typically involving in-house experts and students. In recent years, globalisation has emerged as an important business enabler in higher education (Mayhew et al., 2012) and introduced many situational project allocation scenarios that are not present in conventional circumstances. In such scenarios, universities or campuses within a university may need to leverage expertise of faculty and students outside the university/campus jurisdiction to find feasible project allocations. Interdisciplinary research (Mayhew et al., 2012), Intra-institute and inter-institute research collaborations (Sa, 2008), multi-campus universities (Ebden, 2010), and cross-disciplinary virtual teams (Brewer et al., 2015) are some areas in higher education where such scenarios are pertinent.

Existing SPA solutions are not capable of solving the situational SPA problem, since they have been designed for standalone applications (Teo and Ho, 1998; Pan et al., 2009; 
Anwar and Bahaj, 2003; Moussa and Abu El-Atta, 2011), which are not interoperable and reusable across educational boundaries.

The paper presents a semantic SPA framework that addresses the situational SPA problem, by leveraging existing semantic investments made by the educational institutions. The framework includes:

a a new web ontology language (OWL) ontology called the 'SPA ontology', which standardises the SPA concepts semantically

b SOAP-based Web services that implement the SPA solution

c OWL-S specification, which enables the automatic discovery, composition, and invocation of the web services by educational institutions or semantic agents on the web, for situational SPA scenarios.

The remainder of the paper is organised as follows. Section 2 provides a brief background on the application of semantic Web technologies in education, besides covering the existing approaches to solve the SPA problem. Section 3 presents the Semantic SPA framework and explains about its components. Section 4 presents a case study and compares the semantic SPA framework with existing literature. Lastly, Section 5 concludes the paper with some recommendations for future research.

\section{Background}

The business needs of the education sector have evolved from information sharing in Web 1.0 to user collaboration in Web 2.0 to semantic interoperability in Web 3.0 (Devedzic, 2006). Since the paper proposes a semantic framework to solve the situational SPA problem, first, the current influence of semantic Web technologies on education is reviewed, and subsequently, a brief background of the existing SPA approaches is presented.

\subsection{Semantic web technologies in education}

Semantic Web is an extension of the World Wide Web that facilitates organisations and people to share data across geographical, organisational, and application boundaries (Shadbolt et al., 2006). To facilitate interoperability and data sharing, domain ontologies are developed which conceptually define the data elements within the domain (Shadbolt et al., 2006). Researchers have defined several domain ontologies in higher education, such as ontology to model Web-based e-learning systems for higher education (Bucos et al., 2010), ontology for collaborative learning systems (Barros et al., 2002), DOAP (description of a project) ontology that describes educational and software projects (Apache Software Foundation, 2019).

It is observed that the adoption of semantic technologies in educational domain is undergoing a transition from soft to hard semantic technologies (Tiropanis et al., 2009). While soft technologies express the meaning of educational resources in human-readable formats such as domain ontologies, hard technologies express the resources in machine-processable formats, such as resource description framework (RDF). Some examples of the usage of hard technologies in higher education include annotation and 
search of educational video resources (Yu et al., 2012), LOD for conferences in various disciplines (Springer, 2015), e-learning models (Millard et al., 2008; Ghaleb et al., 2006), teacher education (Czerkawski, 2014), and offer selection process (Kumar et al., 2009).

\subsection{Existing SPA approaches}

Two-sided matching problems (Gale and Shapley, 1962) are a general category of computational problems, having two disjointed sets of participants, A and B, each of whom submits a preference list consisting of participants from the other set. The objective of a two-sided matching problem is to find a stable match between the members of $A$ and members of $B$, subject to preferences and constraints (Abraham et al., 2007), that eventually results in subset $A B$. Furthermore, the stable matching ensures that every element $a_{i}$ in the set $A$ is assigned the best element $b i$ in set $B$, based on the preference. The concept of two-sided matching problems was first demonstrated for college admissions by Gale and Shapley (1962) and later extended to real-life problems, such as 'roommate assignment' problem (Irving, 1985), 'workforce assignment' problem (Gharote et al., 2015), 'student-project allocation (SPA)' problem (Abraham et al., 2007; Teo and Ho, 1998) etc.

In universities, the problem of assigning students to projects, subject to certain constraints and preferences, is referred to as the SPA problem (Teo and Ho, 1998). SPAs can happen in both conventional and situational scenarios. Conventional scenarios involve in-house experts and students from the same discipline for routine project allocations such as the final-year projects (FYPs) (Teo and Ho, 1998). Typically, experts offer a variety of projects, and for those projects, exercise preferences over the students that they are willing to supervise. Likewise, among the offered projects, the students too can exercise their preference for the projects acceptable to them. In addition, various constraints are specified such as project capacity constraint which indicates the maximum number of students that could be assigned to a project and expert capacity constraint which restricts the maximum number of students that an expert can supervise (Abraham et al., 2007).

To solve the conventional SPA problem, early researchers focused on feasibility aspect (Teo and Ho, 1998) and obtained all possible SPAs, which are not necessarily optimal. Later, focusing on the single objective of providing an optimal solution, various techniques, such as integer programming (Anwar and Bahaj, 2003), genetic algorithm (Harper et al., 2005), etc., were developed. In recent years, the SPA problem is perceived to be multi-objective, wherein multiple fringe objectives are added to the basic project allocation objective. Following are some multi-objective solutions:

a linear programming (Abraham et al., 2007), which considers expert-student preferences (expert-orientation) and student-project preferences (student-orientation)

b SPA-P (Manlove and Malley, 2008), which considers expert-project preferences (project- orientation)

c goal programming (Pan et al., 2009), which considers grade point average (GPA) of students

d fuzzy evolutionary approach (Rachmawati and Srinivasan, 2005), which considers a combination of cumulative average point (CAP) and preference list provided by students. 
Furthermore, the correctness of these solutions depends on satisfying two conditions:

1 algorithm terminates with a stable matching

2 student-project pairs deleted during the execution of the algorithm do not form blocking pairs, which disrupt the stability of the algorithm.

Following are some situational SPA scenarios in higher education:

a Researchers in one discipline look for collaboration with researchers in other disciplines to complete research projects (Mayhew et al., 2012).

b Explore feasible student-project-expert combinations involving students, projects, and experts from the available global pool in multi-university campuses, based on skill and talent (Ebden, 2010).

c Experts in one discipline collaborate virtually with geographically dispersed cross-disciplinary student teams to accomplish educational goals, using information and communication technologies (ICTs) (Brewer et al., 2015).

The existing SPA solutions (Teo and Ho, 1998; Pan et al., 2009; Anwar and Bahaj, 2003; Moussa and Abu El-Atta, 2011) are designed as standalone applications, do not support interoperability, and hence cannot be used for solving the situational SPA problem. The authors propose the semantic SPA framework in the next section that addresses the situational SPA problem.

\section{Semantic SPA framework}

The semantic SPA framework comprises of four components namely terminology, SPA ontology, OWL-S specification, and SPA service, as shown in Figure 1. The SPA ontology describes the classes, attributes, relations, and restrictions, which are required to establish semantic relationships between different elements within the SPA problem domain. The SPA service represents a composite web service which implements the SPA solution. The OWL-S specification describes the SPA service semantically through sub-ontologies of SPA profile, SPA process and SPA grounding, which facilitate the automatic discovery, composition, and invocation of the SPA service respectively. Lastly, the terminology provides formal definitions describing the basic elements of the SPA problem and is referenced by all other components of the framework. The framework components are explained in subsequent sections.

\subsection{Terminology}

In this section, the authors redefine the basic elements of the SPA problem using semantic notations. Abraham's lecturer-oriented and student-oriented algorithms (Abraham et al., 2007) are considered as the basis for identifying various elements of the SPA problem.

Definition 1: A situational SPA scenario is defined as an SPA operation (OP), which is a 5-tuple of the form, $\mathrm{OP}=(\mathrm{ON}, \mathrm{E}, \mathrm{P}, \mathrm{S}, \mathrm{G})$, where 
- $\mathrm{ON}$ is the SPA ontology that semantically describes various data elements of the SPA problem

- $\quad E=\left\{e_{1}, e_{2}, \ldots, e_{1}\right\}$ represents a finite set of 1 experts wherein each expert is denoted as a resource having a unique $\mathrm{RDF}$ profile adhering to $\mathrm{ON}$.

- $\quad \mathrm{P}=\left\{\mathrm{p}_{1}, \mathrm{p}_{2}, \ldots, \mathrm{p}_{\mathrm{j}}\right\}$ represents a finite set of $\mathrm{j}$ projects wherein each project is denoted as a resource having a unique $\mathrm{RDF}$ profile adhering to $\mathrm{ON}$.

- $\mathrm{S}=\left\{\mathrm{s}_{1}, \mathrm{~s}_{2}, \ldots, \mathrm{s}_{\mathrm{i}}\right\}$ represents a finite set of I students wherein each student is denoted as a resource having a unique $\mathrm{RDF}$ profile adhering to $\mathrm{ON}$.

- $\mathrm{G}$ is the graph that is constructed during an SPA operation.

The expert/student/ project profiles, constructed during an SPA operation, comprise RDF triples of the form [subject, predicate, object] and are formally defined as a graph $\mathrm{G}$, as follows.

Definition 2: An RDF graph G, constructed during OP, can be defined as $\mathrm{G}=(\mathrm{SU}, \mathrm{PR}, \mathrm{OB})$, wherein $\mathrm{SU}, \mathrm{PR}$, and $\mathrm{OB}$ represent the nodes in the graph

- $\quad \mathrm{SU}=(\mathrm{E}|\mathrm{P}| \mathrm{S})$ represents the subject, where $\mathrm{E}$ is the expert, $\mathrm{P}$ the project, and $\mathrm{S}$ the student.

- $\quad \mathrm{PR}=$ (projectCapacity $\mid$ projectPreference $\mid$ studentPreference $\mid$ projectOffering $\mid$ expertCapacity $\mid$ allocatedTo $\mid$ offerredBy) represents the list of predicates for the SPA problem.

- $\quad \mathrm{OB}=(\mathrm{L} \mid \mathrm{OL})$ represents the list of objects, where $\mathrm{L} \in \mathrm{N}>0$ is a literal value; $\mathrm{OL}$ is a strictly ranked order of objects comprising either students or projects represented as a sequence.

The permissible combinations in which SU, PR, and OB triples appear in graph G during the SPA operation OP are formally defined as vocabulary. The vocabulary follows certain constraints which are represented as assignment. The resulting student-projectexpert allocations are represented as stable matching. Formal definitions for vocabulary, assignment and stable matching are as follows.

Definition 3: Vocabulary VO, assignment AN, and stable matching SM are defined as follows:

- $\quad \mathrm{VO}=((\mathrm{P}$ projectCapacity $\mathrm{L}) \mid(\mathrm{S}$ projectPreference $\mathrm{OL}|\mathrm{OL}| \mathrm{P}) \mid$

(E studentPreference OL $|\mathrm{OL}| \mathrm{S}$ ) (E projectOffering OL $|\mathrm{OL}| \mathrm{P}) \mid$

(E expertCapacity L)) is the vocabulary constructed for Graph G denoting [SU, PR, OB] triples

- $\quad \mathrm{AN} \mid(\mathrm{S} \times \mathrm{P})$ such that:

1 Each pair $\left(s_{i}, p_{j}\right) \in A N$, where $\left\{s_{i} \in S, p_{j} \in P\right\}$ implies that $p_{j}$ is in the preference list of $\mathrm{s}_{\mathrm{i}}$.

2 For each student $\mathrm{s}_{\mathrm{i}} \in \mathrm{S},\left|\left\{\left(\mathrm{s}_{\mathrm{i}}, \mathrm{p}_{\mathrm{j}}\right) \in \mathrm{AN}: \mathrm{p}_{\mathrm{j}} \in \mathrm{P}\right\}\right| \leq 1$. Each student is assigned, at the most, one project in $\mathrm{AN}$.

- $\quad \mathrm{SM}=(\mathrm{S}$ allocatedTo $\mathrm{P}) \mathrm{U}(\mathrm{P}$ offeredBy $\mathrm{E})$ represents the stable matching triples for the SPA operation OP satisfying the following conditions: 
1 For each project $\mathrm{p}_{\mathrm{j}} \in \mathrm{P},\left|\mathrm{AN}\left(\mathrm{p}_{\mathrm{j}}\right)\right| \leq \operatorname{projectCapacity}\left(\mathrm{p}_{\mathrm{j}}\right)$, where $\mathrm{AN}(\mathrm{pj})$ denotes the set of students assigned to $\mathrm{p}_{\mathrm{j}}$ in $\mathrm{AN}$.

2 For each expert el $\in \mathrm{E}$, expert capacity constraint is met such that, the students allocated to el $\leq$ expertCapacity(el)

Figure 1 The semantic SPA framework

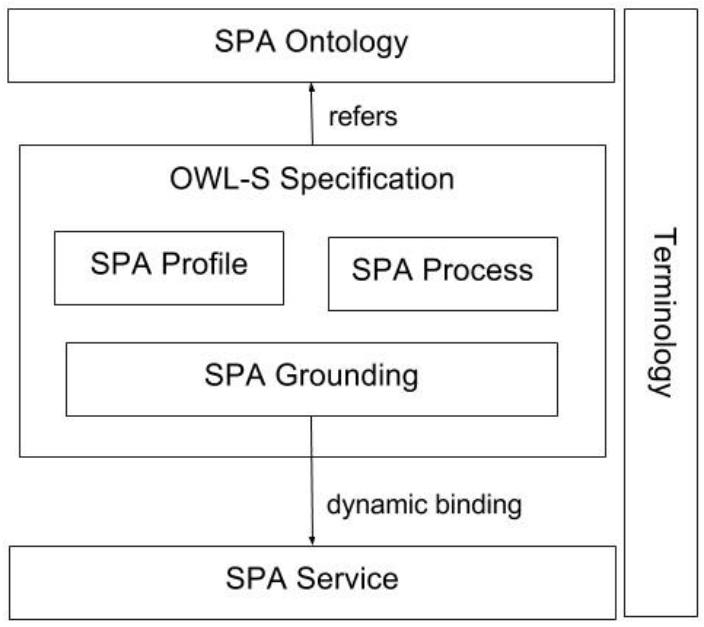

\subsection{SPA ontology}

A new OWL ontology, named the SPA ontology, is created to semantically describe the important elements of the SPA problem in RDF format, whose domain model is shown in Figure 2. From a semantic perspective, the key to framing ontology for the SPA problem involves finding suitable vocabularies that express project, student, and expert information. DOAP is a W3C defined standard vocabulary (Apache Software Foundation, 2019) used to define projects and is used as the base vocabulary to express the project information. The class spa:Project is defined as a subclass of DOAP and inherits all DOAP properties, besides defining projectCapacity, resourceURI, and fundedBy as properties. FOAF is a vocabulary that describes profile information of the concept, known as Person, with properties such as name, phone number, and mail. Since experts and students are basically subclasses of Person class, FOAF is used as the base vocabulary to define expert and student profiles. The class spa:Person inherits from foaf:Person class and defines hasSkill predicate that has association with $c v$ :Skill class, which describes skills of a person. Further, spa:Person and spa:Project classes refer to organisational and location details provided by ORG and GN ontologies respectively.

Correlating with the terms defined in Section 3.1 the E, P, and S tuples represent individuals of the expert, project, and student classes respectively. While the predicates projectCapacity, resourceURI, campusID, etc., represent data properties within the SPA ontology, the predicates hasSkill, allocatedTo, and offerredBy represent the object properties, wherein the domain and range values are defined by classes and not literals, as in the case of data properties. 
Figure 2 The SPA ontology

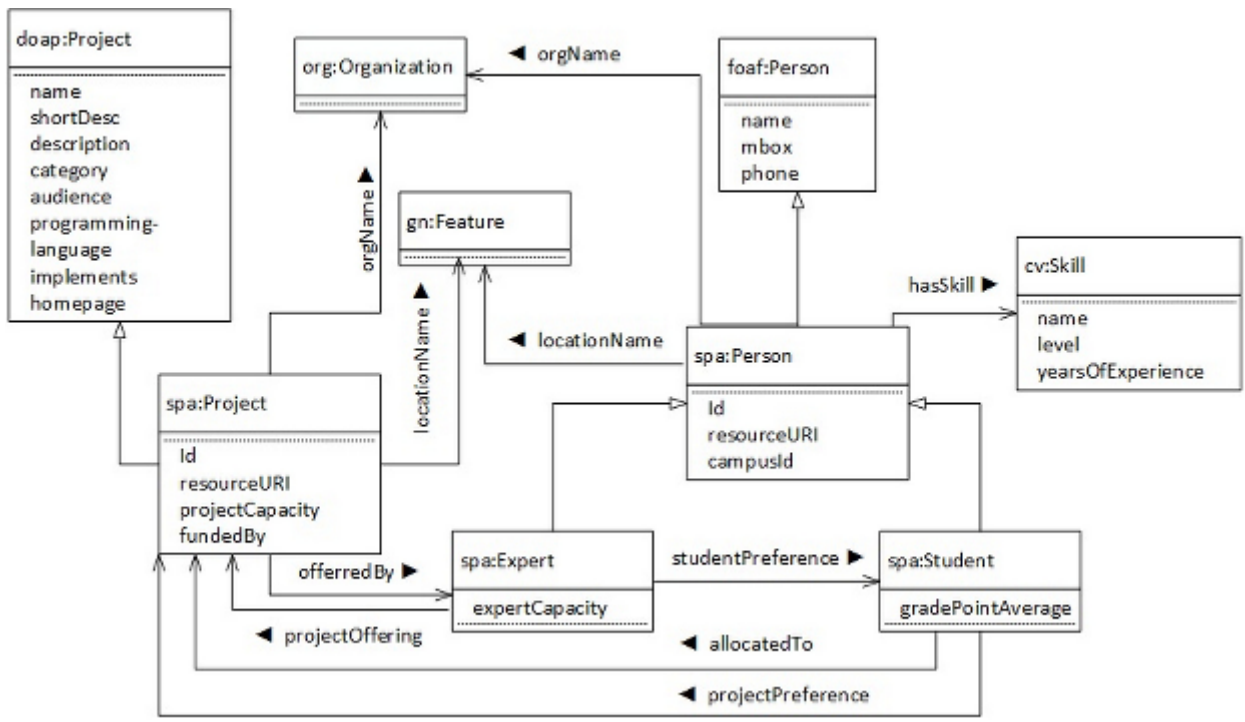

\subsection{SPA service}

Since in any situational SPA scenario, expert availability to execute projects is more vital than student or project availability, the authors have chosen to implement the lecturer-oriented SPA algorithm (Abraham et al., 2007), as a service, called the SPA service. The SPA service is a composite service comprising of three atomic Web services namely ValidateProfile, FilterProfil, and ExecuteSPA, which are developed as SOAP-based WCF (Windows Communication Framework) services in C\# language. The atomic services validate the input RDF profiles, filter the content of the profiles based on specified criteria, and finally execute the lecturer- oriented SPA algorithm, as explained in the following.

\section{ValidateProfile Web service}

The Validate Profile service validates the expert, student, and project profiles for correctness and completeness. The service consists of two operations namely 'ValidateProfileXML' and 'ValidateProfileNT', which validate the RDF profiles in $\mathrm{RDF} / \mathrm{XML}$ and N-Triples formats respectively. The ValidateProfileXML operation accepts the XML profile and the XSD schema (which adheres to the SPA ontology) as input, validates the profile data against the schema and returns validation status as output. The following types of errors are validated:
a malformed syntax
b invalid element types
c incorrect elements
d nil elements
e invalid data. 
The ValidateProfileNT operation accepts the RDF profile in N-Triples format as input, validates the profile, and returns validation status as output.

2 FilterProfile Web service

The service consists of a single method 'FilterProfileOperation' which takes the RDF profile and filter criteria as inputs. The service facilitates the filtering of the RDF profiles based on the specified criteria. The project, expert, and student profiles contain different sets of criteria such as:

a category, technology, audience, programming-language, etc., for projects

b skill name, skill level, years of experience for experts

c GPA, and skill for students.

The criteria for filtering the profile is input as a SPARQL query to the service, which executes the query on the profile, and returns the matching triples as output. The query parameter comprises of two parts: prefix namespace and filter query. First, the service assesses the prefix namespaces within the query for correctness. Second, the service executes the filter query on the RDF profile, creates resultant RDF triples which are enclosed in an RDF graph and returns them as output.

3 ExecuteSPA Web service

The service takes the expert, student, and project profiles as input, and returns the allocation information as a graph in [SU, PR, OB] form, represented as follows:

- $\mathrm{S}_{\mathrm{i}}$ allocatedTo $\mathrm{P}_{\mathrm{j}}$ where $\mathrm{S}_{\mathrm{i}} \in \mathrm{S}$ and $\mathrm{P}_{\mathrm{j}} \in \mathrm{P}$

- $\mathrm{P}_{\mathrm{j}}$ offeredBy $\mathrm{E}_{1}$ where $\mathrm{P}_{\mathrm{j}} \in \mathrm{P}$ and $\mathrm{E}_{1} \in \mathrm{E}$

\subsection{OWL-S specification}

The SPA service is described semantically using the OWL-S specification, which facilitates the automatic discovery, composition, and invocation of the service from either Institutions seeking SPAs or from semantic agents on the Web. The specification comprises of three sub-ontologies namely, SPA profile, SPA process, and SPA grounding. The SPA profile sub-ontology advertises the SPA service by providing a service description and categorises the service under NAICS (North American Industry Classification System category of 'Educational support services', having a profile code of 611710). The description and categorisation assists in the discovery of the service. The SPA process sub-ontology provides the input, output, precondition, and effect (IOPE) details for the three atomic services, and further specifies the orchestration sequence for invoking the services. Sequence conditional types of control constructs are used to define the orchestration sequence, as depicted in Figure 3. As per the orchestration sequence, there are three kinds of invocations:

a sequence of ValidateProfile, FilterProfile, and ExecuteSPA (ideal scenario)

b sequence of ValidateProfile followed by ExecuteSPA, if filtering is not needed

c sequence of FilterProfile followed by ExecuteSPA.

However, validating the profile RDF before the SPA operation is advisable, although optional. Lastly, the SPA grounding sub-ontology provides the mapping between each 
atomic process defined in the OWL-S specification to the corresponding Web service operations.

Figure 3 Orchestration sequence for the SPA service

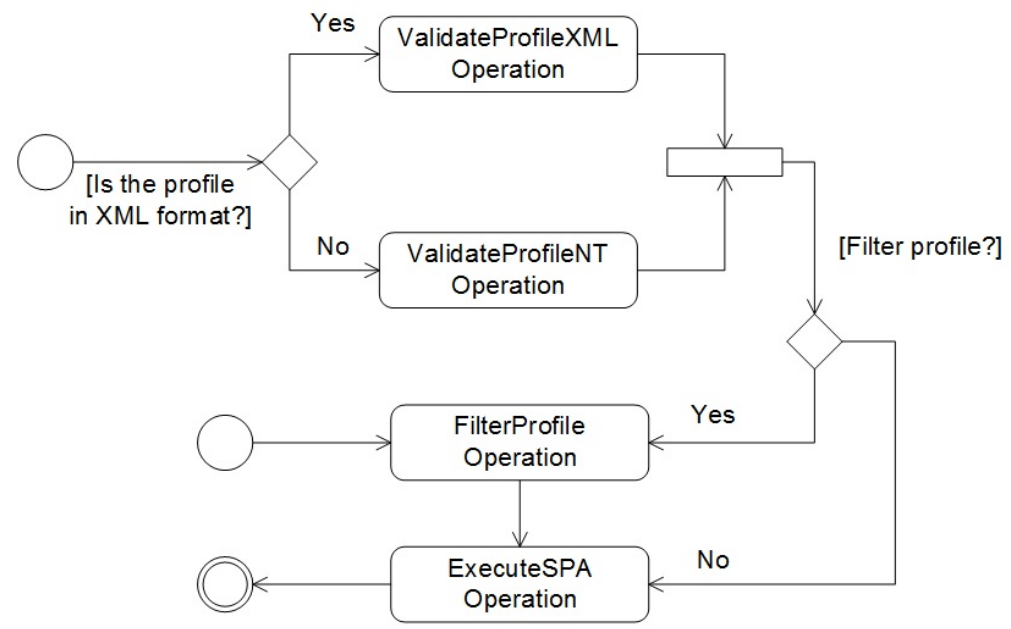

\section{Case study and discussion}

In this section, we demonstrate the application of semantic SPA framework for a hypothetical case based on Abraham's example (Abraham et al., 2007), re-shown in Table 1. The purpose of the case is to demonstrate the automatic discovery, composition, and invocation of the semantic Web services, for a given scenario, and not to mimic a real-world scenario. We have developed a semantic client application using the ASP.NET technology, which facilitates the automatic execution of the situational SPA.

Table 1 Sample SPA instance

\begin{tabular}{lcc}
\hline Student preferences & Expert preferences & Project offerings \\
\hline $\mathrm{s}_{1}: \mathrm{p}_{1}, \mathrm{p}_{2}$ & $\mathrm{e}_{1}: \mathrm{s}_{2}, \mathrm{~s}_{1}, \mathrm{~s}_{3}, \mathrm{~s}_{5}, \mathrm{~s}_{5}$ & $\mathrm{e}_{1}$ offers $\mathrm{p}_{1}, \mathrm{p}_{2}, \mathrm{p}_{3}$ \\
$\mathrm{~s}_{2}: \mathrm{p}_{4}, \mathrm{p}_{1}$ & $\mathrm{e}_{2}: \mathrm{s}_{2}$ & $\mathrm{e}_{2}$ offers $\mathrm{p}_{4}$ \\
$\mathrm{~s}_{3}: \mathrm{p}_{5}, \mathrm{p}_{2}$ & - & \\
$\mathrm{s}_{4}: \mathrm{p}_{3}$ & - & \\
$\mathrm{s}_{5}: \mathrm{p}_{1}, \mathrm{p}_{2}, \mathrm{p}_{3}$ & - & \\
Project capacities: $\left|\mathrm{p}_{1}\right|=1,\left|\mathrm{p}_{2}\right|=1,\left|\mathrm{p}_{3}\right|=1,\left|\mathrm{p}_{4}\right|=1,\left|\mathrm{p}_{5}\right|=1,\left|\mathrm{p}_{6}\right|=1$ & \\
Expert capacities: $\left|\mathrm{e}_{1}\right|=3,\left|\mathrm{e}_{2}\right|=1$ &
\end{tabular}

Source: Abraham et al. (2007)

Input profile formation: The expert, project, and student profiles required for the SPA execution were formed in the following way. Projects, in RDF format, were considered on a random sampling basis from the Apache project store (Apache Software Foundation, 2017). A total of 15 projects were randomly selected from the store. The project profiles were consolidated into $\mathrm{N}$-triples format and validated for correctness using 
'ValidateProfile' service. Next, using the FilterProfile service, top six projects were considered satisfying the criteria:

a programming-language as 'Java'

b category as either 'Cloud' or 'Database'.

In a similar way, 12 faculties (experts) of Manipal Academy of Higher Education, belonging to the departments of Computer Science and Engineering, Information and Computer Technology, and School of Information Science were considered on a random sampling basis, three from each department. The faculty profiles, in RDF format, were taken from the 'EPrints' digital library. Using the FilterProfile service, top two experts were considered satisfying the same filter criteria as the projects, i.e., having Java experience and working in database or Cloud technology. The student profile was framed hypothetically in N-Triples format. The resulting profiles consisted of two experts, five students, and six projects, resembling the instance depicted in Table 1.

- $\quad$ Automatic execution of semantic SPA: The semantic client application took the OWL-S specifications (SPA profile, SPA process and SPA grounding) as inputs, parsed through the process specification and discovered the IOPE for each atomic service and the overall orchestration sequence. Next, using the 'ValidateSPA' service, the student, project, and expert profiles were validated for correctness and completeness. Since the filtering of the profiles was already completed, the 'ExecuteSPA' service was invoked.

- $\quad$ Comparison with existing approaches: Functionality-wise, the proposed framework extends Abraham et al.'s (2007) expert-oriented algorithm, wherein the algorithm's data elements are semantically defined as RDF triples, with the data semantics adhering to the SPA ontology. Further, the proposed framework delivers a multi-objective SPA solution, which encompasses multiple objectives stated in literature (Abraham et al., 2007; Pan et al., 2009; Rachmawati and Srinivasan, 2005). The framework considers the GPA of students prior to allocation, wherein the GPA functions as a filter parameter to the FilterProfile service. This approach contrasts with the goal programming approach (Pan et al., 2009) and fuzzy evolutionary approach (Rachmawati and Srinivasan, 2005), which consider the GPA of students only during allocation. Prior consideration of GPA is advantageous in situational scenarios such as interdisciplinary research, where there is a capacity constraint on the student intake for allocation and only the best students are considered for subsequent stages of allocation.

From a technological perspective, existing solutions for conventional SPA problem, developed in procedural languages (Teo and Ho, 1998), standalone models (Anwar and Bahaj, 2003; Pan et al., 2009), applets (Moussa and Abu El-Atta, 2011), have limitations and cannot be extended to solve globalisation challenges discussed in this paper. Tiropanis et al. (2009) contend that the problem of cross-institutional collaboration can be best solved through adoption of semantic technologies and that of interoperability through transition from soft to hard semantic technologies. In the semantic SPA framework, the SPA ontology is represented in human-readable form (Figure 2), which represents soft semantic technology. Furthermore, all the core SPA elements, such as expert, student, and project, are expressed in RDF format using standard vocabularies 
such as FOAF and DOAP, which is machine-readable and adheres to hard semantic technology format.

\section{Conclusions}

Advancement in Web technology during the past decade has motivated researchers to reexamine and solve classical problems in education using latest technologies. The situational SPA is one such problem which brings with it interoperability challenges due to globalisation of higher education. To address this problem, the authors propose the semantic SPA framework that includes

a the SPA ontology, which semantically standardises different elements of the SPA problem

b the SPA service that implements three atomic services to solve the situational SPA problem

c the OWL-S specification that facilitates educational Institutions and semantic agents to automatically discover, compose, and invoke the SPA service.

A limitation of the framework is that it addresses only the expert-oriented SPA problem.

It is also interesting to consider the design of other variants of the SPA problem. Accordingly, the future work includes

a implementation of 'student-oriented' and 'project-oriented' operations as part of the 'ExecuteSPA' service

b testing of the Semantic SPA framework for a real-world scenario.

\section{References}

Abraham, D.J., Irving, R.W. and Manlove, D.F. (2007) 'Two algorithms for the student-project allocation problem', Journal of Discrete Algorithms, Vol. 5, No. 1, pp.73-90.

Anwar, A.A. and Bahaj, A.H. (2003) 'Student project allocation using integer programming', IEEE Transactions on Education, Vol. 46, No. 3, pp.359-367.

Apache Software Foundation (2017) Apache Projects Directory [online] https://projects.apache.org/projects.html (accessed 29 Jan 2019).

Apache Software Foundation (2019) DOAP Files [online] https://projects.apache.org/doap.html (accessed 5 March 2019).

Barros, B., Verdejo, M.F., Read, T. and Mizoguchi, R. (2002) 'Applications of a collaborative learning ontology', in Proceedings of the Second Mexican International Conference on Artificial Intelligence (MICAI 2002), Mexico, pp.301-310.

Brewer, P.E., Mitchell, A., Sanders, R., Wallace, P. and Wood, D.D. (2015) 'Teaching and learning in cross-disciplinary virtual teams', IEEE Transactions on Professional Communication, Vol. 58, No. 2, pp.208-229.

Bucos, M., Dragulescu, B. and Veltan, M. (2010) 'Designing a semantic web ontology for E-learning in higher education', in Proceedings of 9th International Symposium on Electronics and Telecommunications (ISETC), Timisoara, Romania, pp.415-418.

Czerkawski, B.O. (2014) 'The semantic web in teacher education', The Turkish Online Journal of Educational Technology, Vol. 13, No. 4, pp.144-147. 
Devedzic, V. (2006) Semantic Web and Education, 1st ed., Springer, USA,

Ebden, M. (2010) 'We are on a steep learning curve: the benefits and challenges of multi-campus university course delivery', Research and Development in Higher Education, July, Vol. 33, pp.267-277.

Gale, D. and Shapley, L.S. (1962) 'College admissions and the stability of marriage', American Mathematical Monthly, Vol. 69, No. 1, pp.9-15.

Ghaleb, F. et al. (2006) 'E-learning model based on semantic web technology', International Journal of Computing and Information Sciences, Vol. 4, No. 2, pp.63-71.

Gharote, M., Patil, R., Lodha, S. and Raman, R. (2015) 'Assignment of trainees to software project requirements: a stable matching based approach', Computers and Industrial Engineering, September, Vol. 87, pp.228-237.

Harper, P.R., de Senna, V., Vieira, I.T. and Shahani, A.K. (2005) 'A genetic algorithm for the project assignment problem', Computers and Operations Research, Vol. 32, No. 5, pp.1255-1265.

Irving, R.W. (1985) 'An efficient algorithm for the 'stable roommates' problem', Journal of Algorithms, Vol. 6, No. 4, pp.577-595.

Kumar, S., Kumar, K. and Jain, A. (2009) 'A semantic web technology based framework for educational-offer selection in higher education', International Journal of Metadata, Semantics and Ontologies, Vol. 4, No. 3, pp.165-182.

Linked Universities (2014) [online] http://linkeduniversities.org/lu/index.php/about/index.html (accessed 15 July 2018).

Liu, T. and Kender, J.R. (2004) 'Lecture videos for e-learning: current research and challenges', in Proceedings of Sixth International Symposium on Multimedia Software Engineering, Miami, FL, USA, pp.574-578.

Manlove, D.F. and Malley, G.O. (2008) 'Student-project allocation with preferences over projects', Journal of Discrete Algorithms, Vol. 6, No. 4, pp.553-560.

Mayhew, M.B., Guo, X., Haase, S.B. and Hartemink, A.J. (2012) 'Close encounters of the collaborative kind', IEEE Computer, Vol. 45, No. 3, pp.24-30.

Millard, D. et al. (2008) 'Semantic web services for e-learning', International Journal of Knowledge and Learning, Vol. 4, Nos. 2/3, pp.298-315.

Moussa, M.I. and Abu El-Atta, A.H. (2011) 'A Visual Implementation of student project allocation', International Journal of Computer Theory and Engineering, April, Vol. 3, No. 2, pp.178-184.

Murugesan, S. (2007) 'Understanding Web 2.0', IT Professional, Vol. 9, No. 4, pp.34-41.

Pan, L., Chu, S.C. K., Han, G. and Huang, J.Z. (2009) 'Multi-criteria student project allocation: a case study of goal programming formulation with DSS implementation', in Proceedings of the Eighth International Symposium on Operations Research and Its Applications (ISORA), Zhangjiajie, China, pp.75-82.

Rachmawati, L. and Srinivasan, D. (2005) 'A hybrid fuzzy evolutionary algorithm for a multi-objective resource allocation problem', in Proceedings of Fifth International Conference on Hybrid Intelligent Systems, Rio de Janeiro, Brazil, p.6.

Sa, C.M. (2008) 'Interdisciplinary strategies in U.S. research universities', Higher Education, Vol. 55, No. 5, pp.537-552.

Shadbolt, N., Hall, W. and Berners-Lee, T. (2006) 'The semantic web revisited', IEEE Intelligent Systems, Vol. 21, No. 3, pp.96-101.

Solemon, B., Ariffin, I., Din, M.M. and Anwar, R.M. (2013) 'A review of the uses of crowd-sourcing in higher education', International Journal of Asian Social Science, Vol. 3, No. 9, pp.2066-2073.

Springer LOD (2015) [online] https://www.springer.com/gp/about-springer/media/press-releases/ corporate/springer-starts-pilot-project-on-linked-open-data/51686 (accessed 15 June 2018). 
Teo, C.Y. and Ho, D.J. (1998) 'A systematic approach to the implementation of final year project in an electrical engineering undergraduate course', IEEE Transactions on Education, Vol. 41, No. 1, pp.25-30.

Tiropanis, T., Davis, H., Millard, D. and Weal, M. (2009) 'Semantic technologies for learning and teaching in the Web 2.0 era', IEEE Intelligent Systems, Vol. 24, No. 6, pp.49-53.

Yu, H.Q., Pedrinaci, C., Dietze, S. and Domingue, J. (2012) 'Using linked data to annotate and search educational video resources for supporting distance learning', IEEE Transactions on Learning Technologies, Vol. 5, No. 2, pp.130-142. 